\title{
Esercizio fisico e doping: ricadute in medicina della sessualità
}

\author{
Andrea Sansone $^{1} \cdot$ Anna Schiavo $^{2} \cdot$ Francesco Romanelli $^{2} \cdot$ Emmanuele A. Jannini $^{1}$
}

Accettato: 2 gennaio 2021 / Pubblicato online: 11 agosto 2021

(C) The Author(s) 2021

Sommario L'attività fisica rappresenta uno strumento essenziale per la prevenzione e la tutela della salute individuale. Ciononostante, solo una minoranza degli adulti raggiunge i livelli raccomandati di esercizio fisico. Nell'ambito della sessuologia medica, intervenire sulla sedentarietà può migliorare la salute sessuale e riproduttiva; tuttavia, è necessario che l'attività fisica sia adeguata, onde evitare lo sviluppo di quadri patologici come la triade dell'atleta o l'ipogonadismo indotto da esercizio fisico. Inoltre, l'eventuale uso di "sostanze atte a migliorare l' apparenza e la performance" ha trasceso i confini dello sport agonistico, trovando largo utilizzo fra gli atleti amatoriali al fine di massimizzare la resa dell'esercizio, sebbene siano noti gli effetti avversi sulla salute sessuale e riproduttiva in entrambi i sessi.

Parole chiave Doping - Anabolizzanti - Medicina della sessualità $\cdot$ Esercizio fisico $\cdot$ Salute riproduttiva .

Prevenzione

Proposto da Emanuela Greco.

Informazioni Supplementari La versione online contiene materiale supplementare disponibile su

https://doi.org/10.1007/s40619-021-00930-4.

$\triangle$ A. Sansone

andreasansone85@gmail.com

1 Cattedra di Endocrinologia e Sessuologia Medica (ENDOSEX), Dipartimento di Medicina dei Sistemi, Università di Roma Tor Vergata, Roma, Italia

2 Sezione di Fisiopatologia Medica, Scienza dell' Alimentazione ed Endocrinologia, Dipartimento di Medicina Sperimentale, Sapienza Università di Roma, Roma, Italia

\section{Introduzione}

Un corretto stile di vita deve necessariamente includere una regolare attività fisica: ciononostante, secondo recenti stime dell'Istituto Superiore di Sanità, solo un'esigua minoranza degli italiani svolge regolare attività fisica. Tale fenomeno coinvolge tutte le fasce d'età: si stima che il $90 \%$ degli adolescenti e il 34\% degli adulti tra i 18 e i 69 anni non raggiungano i livelli raccomandati di attività fisica, e anche in età pediatrica il $60 \%$ svolge meno attività fisica al giorno di quanto sia consigliato [1].

I benefici di un'adeguata attività fisica coinvolgono tutti gli apparati e i sistemi: nell'ambito della medicina della sessualità, le ricadute sono spesso trascurate, sebbene siano di chiara rilevanza tanto nell'uomo quanto nella donna. Da un punto di vista meramente antropologico, la performance sportiva può essere paragonata a un indicatore della caratterizzazione sessuale, in misura più evidente nei maschi: difatti, il fenotipo dell'uomo sportivo, con una composizione corporea più definita e una maggior rappresentazione delle masse muscolari, incarna perfettamente gli effetti metabolici del testosterone [2]. Allo stesso modo, la competitività e l'aggressività, caratteristiche necessarie per una competizione sportiva, possono essere a loro volta associate allo stato di androgenizzazione.

Tuttavia, luci e ombre si alternano nell'ambito dell'endocrinologia dello sport. Ai benefici di una corretta attività fisica, difatti, si contrappongono gli effetti avversi dell' $o$ vertraining e dell'uso di sostanze ad azione anabolizzante. L'uso del doping, frequentemente balzato agli onori della cronaca per le note ricadute sulle competizioni agonistiche, non è tuttavia appannaggio degli atleti professionisti: difatti, anche in chi pratica attività fisica ricreazionale è comune il riscontro di agenti ad azione anabolizzante con il solo scopo di migliorare la resa estetica [3]. Tali farmaci possono determinare serie conseguenze per la salute sessuale e 
riproduttiva, e possono contribuire allo sviluppo di vere e proprie condizioni patologiche spesso ignorate dagli stessi atleti [4].

\section{La regolazione endocrina della sessualità}

La funzione sessuale, tanto nel sesso maschile che in quello femminile, è strettamente associata al funzionamento del sistema endocrino. Il testosterone rappresenta chiaramente il principale protagonista della risposta sessuale, contribuendo in larga misura al desiderio sessuale in entrambi i sessi e regolando la funzione erettile; tuttavia, numerosi altri ormoni sono coinvolti nel determinare la funzione sessuale, e il complesso network che ne consegue rappresenta un campo spesso sottostimato e non sempre investigato adeguatamente.

\section{Salute sessuale e riproduttiva maschile}

\section{Esercizio fisico e asse ipotalamo-ipofisi-testicolo}

Come sopra descritto, il testosterone rappresenta il più noto e più importante determinante della salute sessuale maschile e uno dei principali attori nell'esercizio fisico. Il testosterone ha azione trofica sul tessuto muscolare, favorendo l'aumento della massa muscolare e della forza: tuttavia, il ruolo del testosterone si estende oltre questi aspetti, dal momento che effetti positivi sulla composizione corporea, sui processi cognitivi e sul metabolismo glucidico e proteico sono stati frequentemente riportati in letteratura.

Durante l'esercizio acuto (massimale e sub-massimale) i livelli di testosterone vanno incontro a un incremento transitorio. L'esercizio fisico può essere considerato alla stregua di uno stressor esogeno e l'aumento della testosteronemia avrebbe verosimilmente il compito di favorire la risposta omeostatica all'esercizio fisico contribuendo, così, a un miglioramento della performance dell' attività muscolare e a una maggiore capacità funzionale [5]. Tali effetti metabolici del testosterone sono chiaramente apprezzabili nei soggetti ipogonadici in terapia sostitutiva (testosterone replacement therapy, TRT), nei quali un aumento della forza muscolare e della massa magra sono tra i primi riscontri clinici dell'efficacia del trattamento [6]. Ad oggi, i meccanismi coinvolti nell'aumento della testosteronemia durante l'esercizio fisico non sono ancora stati del tutto delucidati: si ritengono plausibili un' aumentata responsività ipofisaria, una migliore funzione delle cellule di Leydig o, ancora, una riduzione nella clearance del testosterone o alterazioni del flusso ematico testicolare.

La risposta endocrina all'esercizio fisico non è univoca: difatti, stimoli diversi per durata, entità e tipologia contribuiscono a dare risposte diverse, come ad esempio osserva- bile nella risposta all'overtraining o a schemi di allenamento inadeguati. Sottoporsi a carichi di esercizio eccessivi può deprimere la funzionalità dell'asse ipotalamo-ipofisi-testicolo, sopprimendo i valori di testosterone in maniera significativa e duratura, seppur spesso mantenendoli all'interno del range fisiologico e senza determinare rialzo compensatorio dei livelli di LH.

Tale quadro clinico e biochimico è stato talora definito exercise-hypogonadal male condition e i potenziali effetti (deleteri o benefici) di una terapia sostitutiva non sono ancora stati del tutto chiariti. Diversi meccanismi patogenetici sono stati ipotizzati alla base di questo fenomeno, quali la resistenza ipofisaria allo stimolo del $\mathrm{GnRH}$ o un'iperprolattinemia transitoria o, ancora, un effetto di feedback negativo ad opera della ghrelina e della leptina. Ulteriore difficoltà allo studio di questo fenomeno deriva dall'impossibilità a "standardizzare" le caratteristiche dell'esercizio fisico, come la durata, la tipologia di esercizio e l'ampiezza dello stimolo. Ciononostante, numerosi studi hanno fornito prove a supporto di un calo delle concentrazioni di testosterone totale e libero, di ridotta pulsatilità della secrezione di FSH e LH, e di aumentati livelli di SHBG in relazione all'esercizio fisico intenso, sia di breve che di lunga durata.

\section{Sport, salute sessuale e fertilità maschile}

La sedentarietà è a tutti gli effetti un fattore di rischio per numerose patologie, incluse le disfunzioni sessuali maschili; non è pertanto sorprendente che un'adeguata attività fisica sia fondamentale per la salute sessuale. Gli interventi sugli stili di vita, compresa la sedentarietà, sono considerati terapia di prima linea per la disfunzione erettile: svolgere adeguato esercizio fisico favorisce la biodisponibilità dell'ossido nitrico e riduce l'espressione delle chemochine infiammatorie TNF- $\alpha$, IL-1 $\beta$ e IL-6 migliorando, quindi, la funzione endoteliale [2]. Una corretta attività fisica sembra, inoltre, in grado di aumentare le concentrazioni di FSH, LH e testosterone, senza raggiungere concentrazioni soprafisiologiche. Tuttavia, nelle forme di ipogonadismo indotto da esercizio fisico, è lo stesso sovraccarico di intensità a favorire il calo del testosterone, che determina disfunzioni sessuali e riduzione della libido [2].

Il ruolo dell'esercizio fisico nella fertilità maschile appare similmente controverso. Un adeguato esercizio fisico è associato a miglior qualità del DNA nemaspermico e a una minore concentrazione di biomarcatori di infiammazione nel liquido seminale; tuttavia, esercizi di carico eccessivo possono tradursi in un peggioramento della qualità seminale $\mathrm{e}$ in un aumento dello stress ossidativo. Oltre al già citato ipogonadismo indotto da esercizio fisico, che pur coinvolge una minoranza degli atleti, altri possibili effetti nocivi dell'esercizio fisico sulla fertilità maschile sono stati ipotizzati come, ad esempio, l'ipossia a carico della circolazione testicolare 


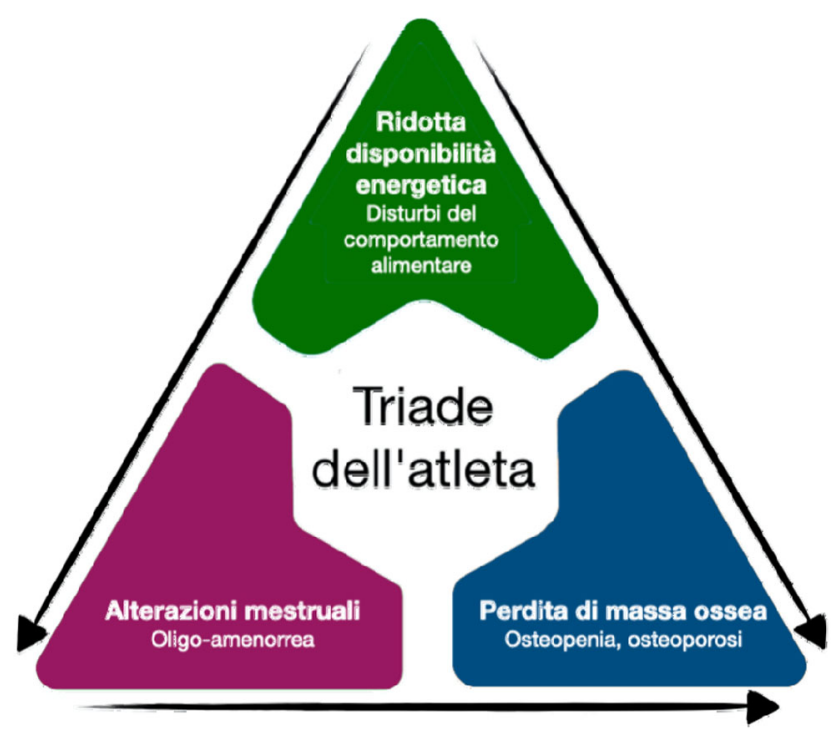

Fig. 1 La triade dell'atleta (Female Athlete Triad)

o l'aumento della temperatura scrotale e della pressione sul piano pelvico in caso di alcuni sport, come ad esempio nel ciclismo. Gli effetti dell'esercizio fisico su altri outcome di fertilità, come ad esempio il tasso di gravidanza clinica o di nati vivi, non sono stati adeguatamente studiati finora.

\section{Salute sessuale e riproduttiva femminile}

\section{Esercizio fisico e asse ipotalamo-ipofisi-ovaio}

Gli effetti dell'attività fisica sull'assetto ormonale femminile e sulla funzionalità ovarica sono difficilmente standardizzabili, dal momento che diverse variabili individuali (età della donna, regolarità mestruale, peso, abitudini alimentari, PCOS, gravidanza, ecc.) e proprie del tipo di attività (tipo di esercizio, durata, intensità dell'allenamento) influenzano la risposta all'esercizio. In particolare, e in misura ancora più evidente di quanto riscontrato nel sesso maschile, un carico eccessivo di attività fisica può indurre una soppressione dell'asse ipotalamo-ipofisi-ovaio, con conseguente ipoestrogenismo e compromissione della funzionalità gonadica [7]. Alla base di tale meccanismo sembra esserci la mutuale modulazione tra sistema endocrino e assetto metabolicoenergetico dell'organismo. Questa interazione si esplica più livelli e, quando intaccata, come avviene ad esempio se si è sottoposti ad alti carichi di lavoro, in termini di intensità o durata, può esitare nella cosiddetta "triade dell'atleta" (Fig. 1). Si tratta di una sindrome clinica, di frequente riscontro nella popolazione atletica femminile, caratterizzata da scarsa disponibilità di energia (più o meno associata a disturbi del comportamento alimentare), disturbi del ciclo mestruale e ridotta densità minerale ossea. L'esercizio fisico troppo intenso, accompagnato da un inadeguato introito calorico giornaliero, induce infatti uno stato carenziale a cui l'organismo reagisce con meccanismi di compenso volti a preservare le funzioni vitali, trascurando quindi sistemi non primariamente coinvolti nella sopravvivenza. Questo pone ovviamente le basi perché tali alterazioni vadano a strutturare condizioni morbose croniche (amenorrea, osteoporosi, DCA), con impatto negativo sulla salute generale e riproduttiva della donna $[4,8]$. In tempi più recenti, questa condizione è anche stata definita con l'acronimo RED-S (relative energy deficiency in sports, deficit relativo di energia nello sport), allo scopo di estenderne l'applicazione anche al genere maschile [9].

Tra i disturbi del ciclo mestruale di più frequente riscontro tra le atlete spicca l'amenorrea di origine ipotalamica che in questa fetta di popolazione, insieme all'oligomenorrea, ha una prevalenza stimata fino al 40\% [10]. Si possono riscontrare, inoltre, anovulazione e deficit luteinico, in percentuale maggiore rispetto a donne di pari età con stile di vita sedentario.

Dal punto di vista ormonale sono state studiate le modificazioni dell' assetto ormonale in risposta all'esercizio fisico, e come queste differiscano in donne allenate e sedentarie [7]. La presenza o meno di bilancio energetico negativo risulta determinante nel modulare la risposta ormonale all'esercizio. Si può notare, dopo esercizio fisico aerobico, una tendenza all'abbassamento dei valori di LH e FSH. Un'intensa attività fisica sembra correlarsi anche a un'alterazione della normale secrezione di GH e dell'asse tiroideo, con una riduzione media dei valori di $\mathrm{T} 3$, interpretabile come forma di compenso in caso di bilancio energetico negativo, con lo scopo di ridurre il consumo calorico. Da segnalare anche il ruolo di alcuni peptidi, quali leptina, grelina, NPY, che, attraverso la loro azione centrale su ipotalamo e ipofisi, ma anche periferica direttamente sull'ovaio, possono modulare negativamente la funzionalità gonadica in risposta a modificazioni della disponibilità energetica e della massa grassa [7]. È ormai ipotesi accreditata che il tessuto adiposo e la massa grassa giochino un ruolo centrale nella regolazione della funzionalità gonadica, grazie alla produzione di adipochine in grado di influenzare la produzione ormonale: è quindi necessario il mantenimento di una soglia critica di massa grassa per una normale attività dell'asse ipotalamo-ipofisi-ovaio.

\section{Sport, salute sessuale e fertilità femminile}

Esattamente come avviene nell'uomo, un equilibrato regime di attività fisica ha indubbi effetti positivi sulla salute sessuale femminile. Contribuisce infatti al miglioramento dello stato di benessere generale e gioca un ruolo importante nel favorire il desiderio e il benessere sessuale, entrambi fattori potentemente influenzati dallo stato psicologico della donna. Anche in questo caso però, l'esercizio fisico non misurato 
Tabella 1 Sostanze ad azione "dopante" e ricadute sulla salute sessuale e riproduttiva maschile. Adattata da [19]

\begin{tabular}{|c|c|c|c|c|c|}
\hline \multirow[t]{2}{*}{ Sostanze } & \multirow[t]{2}{*}{ Usata per: } & \multicolumn{4}{|l|}{ Effetti su: } \\
\hline & & Desiderio sessuale & Erezione & Funzione eiaculatoria & Fertilità \\
\hline $\begin{array}{l}\text { Androgeni } \\
\text { anabolizzanti }\end{array}$ & $\begin{array}{l}\text { - azioni ergogeniche e anabolizzanti } \\
\text { - effetti sul SNC ( } \uparrow \text { aggressività, } \uparrow \\
\text { competitività, ecc.) }\end{array}$ & $\uparrow o \downarrow$ & $\downarrow$ & $\begin{array}{l}\downarrow \text { (eiaculazione ritardata, } \\
\text { aneiaculazione) }\end{array}$ & $\downarrow$ \\
\hline$\beta$-bloccanti & $\begin{array}{l}-\downarrow \text { ansia } \\
-\downarrow \text { tremori } \\
-\downarrow \text { frequenza cardiaca }\end{array}$ & & $\downarrow$ & $\begin{array}{l}\downarrow \text { (eiaculazione ritardata, } \\
\text { aneiaculazione) }\end{array}$ & \\
\hline Diuretici & $\begin{array}{l}\text { - mascherare assunzione sostanze } \\
\text { - definire masse muscolari }\end{array}$ & & $\downarrow$ & $\begin{array}{l}\downarrow \text { (eiaculazione ritardata, } \\
\text { aneiaculazione) }\end{array}$ & \\
\hline $\begin{array}{l}\text { Amfetamine, } \\
\text { stimolanti }\end{array}$ & $\begin{array}{l}\text { - effetti sul SNC ( } \uparrow \text { aggressività, } \uparrow \\
\text { competitività, ecc.) } \\
\text { - azioni ergogeniche (non provate) }\end{array}$ & $\uparrow o \downarrow$ & $\downarrow$ & $\begin{array}{l}\uparrow o \downarrow \text { (eiaculazione precoce } \\
\text { o ritardata, aneiaculazione) }\end{array}$ & $\downarrow$ \\
\hline
\end{tabular}

può rappresentare un fattore di rischio per la salute sessuale, specialmente in alcuni tipi di discipline sportive. Sono frequentemente riscontrate disfunzioni sessuali tra le atlete di sesso femminile che hanno, rispetto alla popolazione generale, un rischio aumentato di sviluppare disturbi del pavimento pelvico. Tali alterazioni possono instaurarsi su base traumatica o cronica in soggetti che praticano sport in cui si favorisce di frequente l'aumento della pressione endoaddominale e/o compressione del pavimento pelvico (es. ciclismo). Questi meccanismi possono influenzare il normale afflusso di sangue e la conduzione nervosa a livello vaginale e clitorideo e indurre lo sviluppo di dispareunia, ridotta lubrificazione vaginale, anorgasmia [11]. Inoltre, le già descritte alterazioni dell' assetto ormonale, secondarie a carichi eccessivi di sforzo fisico, possono influenzare negativamente la funzionalità sessuale della donna in termini di desiderio e disfunzioni sessuali.

Per quanto riguarda la salute riproduttiva della donna, anche in questo caso, l'attività fisica moderata sembra avere un impatto positivo sulla fertilità femminile e sulla salute della prole. Uno stile di vita fisicamente attivo è infatti alla base della prevenzione di patologie metaboliche e cardiovascolari e contribuisce, quindi, ad aumentare la probabilità di gravidanza.

Il rovescio della medaglia è nuovamente rappresentato dell'esercizio fisico non misurato. Tra i meccanismi alla base dei problemi riproduttivi a cui la popolazione di atlete può andare incontro, un ruolo predominante è rivestito dalle già citate disfunzioni mestruali indotte dall'esercizio fisico che possono comportare anovulazione o amenorrea, pregiudicando le possibilità di concepimento anche nel lungo periodo. Spesso, infatti, la regolarità mestruale non viene recuperata nonostante il ripristino di un normale indice di massa corporea e la cessazione dell'inadeguata attività fisica.

\section{Doping, salute sessuale e fertilità}

L'uso di sostanze atte a migliorare l'apparenza e la performance (appearance and performance-enhancing drugs, APED) rappresenta un fenomeno sociale che ha trasceso i confini dello sport d'élite e ha raggiunto dimensioni quasi epidemiche. Molti atleti amatoriali fanno ricorso a sostanze atte a migliorare la resa dell'esercizio fisico, senza considerare i potenziali effetti negativi derivanti dal loro uso [12]. Sebbene nell'immaginario collettivo gli androgeni rivestano il ruolo principe nell' ambito del doping, numerose altre sostanze sono proibite dalla World Anti-Doping Agency (WADA) per il loro potenziale uso come agenti dopanti o come "mascheranti" (Tabella 1). L'assunzione a finalità dopanti è chiaramente diversa dall'uso a scopo terapeutico, per il quale è necessaria un' autorizzazione preventiva all'uso (therapeutic use exemption, TUE) [13] nel caso di atleti affetti da patologie che necessitino di tali trattamenti.

\section{Effetti del doping sulla salute sessuale e riproduttiva maschile}

Gli androgeni anabolizzanti sopprimono l'asse ipotalamoipofisi-testicolo, favorendo lo sviluppo di un ipogonadismo ipogonadotropo le cui manifestazioni più precoci sono il calo del desiderio, la disfunzione erettile e l'infertilità. In particolare, i soggetti che fanno uso di doping sviluppano comunemente disfunzione erettile nel "dopo-ciclo", cioè nel momento in cui i valori ematici di testosterone raggiungono il nadir. Il recupero della funzione erettile dopo il doping può richiedere mesi o anni ed è verosimile che a terapie più "massive" corrisponda un maggior tempo per il recupero. Per facilitare il ritorno a uno stato di eugonadismo è comune il ricorso a idonee terapie farmacologiche, generalmente modulatori selettivi del recettore degli estrogeni (SERM) e 
gonadotropine $[14,15]$ ma la risposta è altamente variabile in base alla durata dell'assunzione degli agenti dopanti e allo schema terapeutico usato. L'uso di diverse molecole (una procedura definita stacking) rappresenta un ostacolo non solo al trattamento, ma anche alla valutazione degli effetti clinici.

Per quanto riguarda la fertilità, si stima che circa il $2 \%$ dei casi di infertilità maschile trovino un fattore causale nell'uso di androgeni. Difatti, il calo dei valori di FSH derivante dalla soppressione dell'asse ipotalamo-ipofisi-testicolo determina un ridotto stimolo alla spermatogenesi che, nei casi di assunzione più prolungata nel tempo, può determinare anche atrofia tubulare e ipotrofia testicolare. Tale fenomeno, tuttavia, va più frequentemente incontro a risoluzione spontanea rispetto alla perdita della funzione erettile [4].

Una menzione a parte meritano tutti quei composti che, pur privi di attività androgenica (o, più in generale, ormonosimile) trovano ampio uso al fine di mascherare l'assunzione di prodotti proibiti o per migliorare performance sportive che non trarrebbero vantaggio dall'uso di anabolizzanti: ad esempio i diuretici, usati per eliminare tracce dell' assunzione di prodotti dopanti, ma anche per definire la massa nel culturismo, o i $\beta$-bloccanti, usati per limitare ansie e tremori negli sport di precisione [4]. L'assunzione di alcuni di questi farmaci può contribuire a favorire lo sviluppo di una disfunzione sessuale o a rendere manifesta una disfunzione altrimenti subclinica [2]. Anche alcuni stimolanti, quali ad esempio le amfetamine, rientrano fra le sostanze assunte allo scopo di migliorare la performance sportiva, per gli effetti sui riflessi, sulla competitività e sulla risposta muscolare; sul piano della sessualità, l'assunzione di questi farmaci può alternativamente migliorare o peggiorare la funzione sessuale, il desiderio e la latenza eiaculatoria [4].

\section{Effetti del doping sulla salute sessuale e riproduttiva femminile}

Come per la controparte maschile, anche nell'ambito degli sport femminili sta aumentando esponenzialmente il ricorso a sostanze proibite per migliorare la performance sportiva. Questo fenomeno è particolarmente frequente in quelle discipline sportive in cui l'aumento di massa muscolare può fornire un vantaggio competitivo. Oltre alle già citate sostanze ad azione non ormonale (diuretici, betabloccanti, integratori di varia natura), si assiste a un sempre più vasto utilizzo di sostanze quali GH, IGF1, insulina che agiscono favorendo l'uptake di glucosio da parte del tessuto muscolare e la sintesi proteica, incrementando la massa muscolare riducendo al contempo la deposizione di massa grassa [16].

Tra i maggiori interferenti la funzione sessuale e riproduttiva delle atlete vanno ovviamente considerati gli steroidi anabolizzanti (AASs), il cui abuso sta aumentando anche nella popolazione femminile, con una prevalenza fino all' $1,6 \%$ [17].
Tabella 2 Conseguenze dell'assunzione di androgeni anabolizzanti sulla salute sessuale e riproduttiva maschile e femminile. Adattata da [18]

\begin{tabular}{ll}
\hline Sesso maschile & Soppressione dell'asse ipotalamo-ipofisi-gonadi: \\
& - Riduzione delle gonadotropine \\
& - Inibizione della spermatogenesi \\
& - Ipotrofia testicolare \\
& - Infertilità \\
& Alopecia \\
& Ginecomastia \\
& Calo della libido \\
& Disfunzione erettile \\
& Sudorazione profusa \\
& Strie cutanee (smagliature) \\
& Acne \\
& Ipogonadismo indotto da androgeni \\
& Soppressione dell'asse ipotalamo-ipofisi-gonadi: \\
& - Riduzione delle gonadotropine \\
& - Anovulazione e amenorrea \\
& - Dismenorrea \\
& - Infertilità \\
Irsutismo e alopecia & Atrofia mammaria \\
& Strie cutanee (smagliature) \\
& Acne \\
Ipertrofia clitoridea \\
Sudorazione profusa \\
Disfonia, alterazione del timbro vocale \\
\hline
\end{tabular}

L'assunzione cronica di queste sostanze induce una soppressione dell' asse ipotalamo-ipofisi-ovaio, sia a livello ipotalamico che ipofisario con diversi meccanismi, che esita in una riduzione di secrezione di gonadotropine e, conseguentemente, di estrogeni [15], con le immaginabili ricadute sulla funzione sessuale e riproduttiva (Tabella 2) [18]. Gli effetti anabolizzanti degli AASs, infatti, non sono scindibili dagli effetti androgenizzanti, con conseguente disfunzione della normale sessualità della donna e della capacità riproduttiva [15]. Si può fare ricorso a svariate molecole e formulazioni (orale, transdermica, iniettiva); la scelta, tra le atlete, è di solito maggiormente orientata sull'oxandrolone, che sembra avere effetti virilizzanti ridotti nella donna rispetto ad altre forme, o su precursori del testosterone quali androstenedione e DHEA, la cui assunzione ha dimostrato un impatto più decisivo sul metabolismo femminile che maschile. Gli effetti avversi possono manifestarsi in una forma dose- e tempo di assunzione-dipendente e includono alterazioni mestruali e mascolinizzazione [15, 18]. Le alterazioni mestruali, secondarie alla soppressione della produzione estrogenica, comprendono menarca ritardato, dismenorrea, oligomenorrea fino all' amenorrea secondaria con anovulazione $[15,18]$. 
Queste pongono ovviamente le basi per disturbi della fertilità, considerando che il tempo di ripresa della normale pulsatilità gonadotropinica, dopo cessazione di abuso, è estremamente variabile, da settimane e mesi [15, 18]. Gli effetti virilizzanti possono manifestarsi a diversi livelli; segni caratteristici sono alopecia e irsutismo, acne, atrofia mammaria, clitoridomegalia e abbassamento del tono di voce con raucedine [18]. Questi ultimi due, al contrario dei precedenti, non sono sempre reversibili con la cessazione di assunzione di AASs. Tutto ciò si traduce spesso in disfunzioni sessuali secondarie a ipoestrogenismo (es. ridotta lubrificazione vaginale) e calo della libido su base ormonale e psicologica; tra gli effetti di tali sostanze si annoverano, infatti, anche alterazioni comportamentali e umorali che vanno dall'aumento di aggressività, in molti casi funzionale alla competizione sportiva, fino a stati depressivi più o meno conclamati [4].

\section{Conclusioni}

L'inquadramento clinico di un paziente non può prescindere dalla valutazione dell' attività fisica: difatti, l'esercizio fisico rappresenta un ottimo strumento di prevenzione e tutela della salute. Questo vale anche nell' ambito della medicina della sessualità, dal momento che esiste una forte connessione fra il grado di attività fisica e la salute sessuale e riproduttiva. Tuttavia, è opportuno ricordare come un'attività fisica inadeguata possa portare all'insorgenza di quadri clinici patologici, come chiaramente manifestato nella triade dell'atleta o nelle forme di ipogonadismo indotto dall'esercizio fisico. Inoltre, è fondamentale approfondire sempre l'eventuale uso di sostanze a scopo di doping: sebbene nel mondo dell'agonismo tali sostanze siano usate per migliorare illecitamente la performance sportiva, è sempre più frequente il ricorso a tali sostanze tra gli sportivi amatoriali a scopo di migliorare l'aspetto fisico. Le potenziali ricadute sulla sfera della medicina della sessualità sono in larga misura imprevedibili, dipendenti dalla tipologia di sostanze usate e dalla loro dose totale e, potenzialmente, addirittura irreversibili.

Funding Note Open access funding provided by Università degli Studi di Roma Tor Vergata within the CRUI-CARE Agreement.

Conflitto di interesse Gli autori Andrea Sansone, Anna Schiavo, Francesco Romanelli e Emmanuele A. Jannini dichiarano di non avere conflitti di interesse.

Consenso informato Lo studio presentato in questo articolo non ha richiesto sperimentazione umana e il consenso sul trattamento dei dati è stato acquisito preliminarmente.

Studi sugli animali Gli autori di questo articolo non hanno eseguito studi sugli animali.
Nota della casa editrice Springer Nature rimane neutrale in riguardo alle rivendicazioni giurisdizionali nelle mappe pubblicate e nelle affiliazioni istituzionali.

Open Access This article is licensed under a Creative Commons Attribution 4.0 International License, which permits use, sharing, adaptation, distribution and reproduction in any medium or format, as long as you give appropriate credit to the original author(s) and the source, provide a link to the Creative Commons licence, and indicate if changes were made. The images or other third party material in this article are included in the article's Creative Commons licence, unless indicated otherwise in a credit line to the material. If material is not included in the article's Creative Commons licence and your intended use is not permitted by statutory regulation or exceeds the permitted use, you will need to obtain permission directly from the copyright holder. To view a copy of this licence, visit http://creativecommons.org/licenses/by/4.0/.

\section{Bibliografia}

1. Istituto Superiore di Sanità (2020) Attività fisica. https://www. issalute.it/index.php/la-salute-dalla-a-alla-z-menu/a/attivitafisica. Accessed 1 December 2020

2. Sgrò P, Di Luigi L (2017) Sport and male sexuality. J Endocrinol Invest 40(9):911-923

3. Lazuras L, Barkoukis V, Loukovitis A et al (2017) "I want it all, and I want it now": lifetime prevalence and reasons for using and abstaining from controlled performance and appearance enhancing substances (PAES) among young exercisers and amateur athletes in five European countries. Front Psychol 8:717

4. Romanelli F, Sansone A, Sgrò P et al (2015) Salute sessuale maschile e femminile, attività motoria e sport. Endocrinologo 16(4):160-166

5. Jannini EA, Moretti C, Fabbri A et al (1988) In: Neuroendocrinologia dello stress. Caleidoscopio italiano. Medical systems, Genoa

6. Saad F, Aversa A, Isidori AM et al (2011) Onset of effects of testosterone treatment and time span until maximum effects are achieved. Eur J Endocrinol 165(5):675-685

7. La Vignera S, Condorelli RA, Cannarella R et al (2018) Sport, doping and female fertility. Reprod Biol Endocrinol 16(1):108

8. De Souza MJ, Nattiv A, Joy E et al (2014) 2014 Female Athlete Triad Coalition consensus statement on treatment and return to play of the female athlete triad: 1st International Conference held in San Francisco, CA, May 2012, and 2nd International Conference held in Indianapolis, IN, May 2013. Clin J Sport Med 24(2):96-119

9. Mountjoy M, Sundgot-Borgen J, Burke L et al (2018) International Olympic Committee (IOC) consensus statement on relative energy deficiency in sport (RED-S): 2018 update. Int J Sport Nutr Exerc Metab 28(4):316-331

10. Scheid JL, De Souza MJ (2010) Menstrual irregularities and energy deficiency in physically active women: the role of ghrelin, PYY and adipocytokines. Med Sport Sci 55:82-102

11. Partin SN, Connell KA, Schrader SM, Guess MK (2014) Les lanternes rouges: the race for information about cycling-related female sexual dysfunction. J Sex Med 11(8):2039-2047

12. Piacentino D, Kotzalidis GD, Del Casale A et al (2015) Anabolicandrogenic steroid use and psychopathology in athletes. A systematic review. Curr Neuropharmacol 13(1):101-121

13. Di Luigi L, Pigozzi F, Sgro P et al (2020) The use of prohibited substances for therapeutic reasons in athletes affected by endocrine diseases and disorders: the therapeutic use exemption (TUE) in clinical endocrinology. J Endocrinol Invest 43(5):563-573 
14. Rahnema CD, Lipshultz LI, Crosnoe LE et al (2014) Anabolic steroid-induced hypogonadism: diagnosis and treatment. Fertil Steril 101(5):1271-1279

15. Anawalt BD (2019) Diagnosis and management of anabolic androgenic steroid use. J Clin Endocrinol Metab 104(7):2490-2500

16. Albertson TE, Chenoweth JA, Colby DK, Sutter ME (2016) The changing drug culture: use and misuse of appearance- and performance-enhancing drugs. FP Essent 441:30-43

17. Sagoe D, Molde H, Andreassen CS et al (2014) The global epidemiology of anabolic-androgenic steroid use: a meta-analysis and meta-regression analysis. Ann Epidemiol 24(5):383-398
18. Nieschlag E, Vorona E (2015) Mechanisms in endocrinology: medical consequences of doping with anabolic androgenic steroids: effects on reproductive functions. Eur J Endocrinol 173(2):R47-R58

19. Sansone A, Sansone M, Vaamonde D et al (2018) Sport, doping and male fertility. Reprod Biol Endocrinol 16(1):114 